\title{
Review Article \\ Optical Transitions and Charge-Exchange in Highly Charged Quasi-Molecules
}

\author{
A. Devdariani, ${ }^{1}$ E. Dalimier, ${ }^{2}$ and P. Sauvan ${ }^{3}$ \\ ${ }^{1}$ Department of Optics and Spectroscopy, St. Petersburg University, St. Petersburg 198904, Russia \\ ${ }^{2}$ Laboratoire pour l'Utilisation des Lasers Intenses LULI, UMR 7605, CNRS-CEA-Ecole Polytechnique-Université Paris 6, \\ UPMC case 128, 4 Place Jussieu, 75252 Paris Cedex 05, France \\ ${ }^{3}$ Departamento de Ingeniería Energética, E.T.S. Ingenieros Industriales, UNED, C/ Juan del Rosal 12, 28040 Madrid, Spain
}

Correspondence should be addressed to E. Dalimier, elisabeth.dalimier@upmc.fr

Received 28 April 2009; Accepted 23 June 2009

Academic Editor: Roland Stamm

Copyright ( 2010 A. Devdariani et al. This is an open access article distributed under the Creative Commons Attribution License, which permits unrestricted use, distribution, and reproduction in any medium, provided the original work is properly cited.

The interaction between quasimolecular states produces not only nonadiabatic transitions but also some exotic features in the wings of the spectral profiles emitted by the ions in collision. Although this concept has been fruitfully used for neutral species, some new highlighted experimental data on quasimolecular optical transitions in hot dense plasma have renewed the interest to the concept in the recent years. The present review deals with highly charged quasimolecules and it is dedicated specifically to quasimolecules formed by two bare nuclei and one bound electron. The reason for this choice is that, for such quasimolecules, the energy terms and the dipole moments of the optical transitions can be obtained straightforwardly in nonrelativistic case without any approximation that are typical for neutrals. Although the results obtained in the frame of the approach developed here are directly applicable to the case of single collisions or to low-density plasmas, they form a reasonable initial approximation for the problem of optical profiles in hot dense plasmas and can be regarded as a safe framework for qualitative discussions of profiles in those environments.

\section{Introduction: Features of Spectral Profiles following the Interaction of Quasimolecular States}

The concept of quasi-molecular (QM) states that are formed during collisions of atomic particles has proved to be successful in describing collision processes at low energies including charge-exchange processes. In frame of the QM approach, nonadiabatic transitions in collisions can be regarded as a consequence of the interaction between different QM states at some interatomic distances and can be analytically described by the Landau-Zener model or the Demkov-Nikitin model $[1,2]$. However the QM states participating in collision processes are very often subjected to optical transitions. The question therefore arises whether the interaction of the states during a collision affects the spectral line shape and if so, how? An analysis based on the general theory of quasistationary states, that is, the states that are unstable due to the emission of photons or electrons during the collisions, has shown that the interaction of QM states does indeed give rise to exotic features in the profiles [3, 4]. In particular, analytical formulae have been obtained describing the features produced by the crossing of QM energy terms $[5,6]$. It should be stressed that the approach proposed here has related the cross-sections of nonadiabatic transitions to the spectral features averaged over impact parameters and Maxwell's distribution.

In spite of successful applications of the elaborated theory to experiments with neutrals, for example, [6-9], attention had not been paid to ion collisions in plasma, until recent experiments in hot dense plasmas [10-14] exhibiting fine structures, that is, satellites in F Lyman $\beta$ profile emitted by a fluorine plasma, dips, and bumps in the $A l$ Lyman $\gamma$ profile emitted by a heterogeneous $A l-C$ plasma. The fine structures in $A l$ Lyman $\gamma$ have been connected to intersections of $\mathrm{QM} \mathrm{Al-C}$ energy terms. This work was the first to reveal charge-exchange signature in the spectral line shape in hot dense plasmas. 
The main objective of the present paper is to overview the recent theoretical and numerical developments in the specific field of spectral signatures of nonadiabatic transitions, namely, charge-exchange processes. Those processes are basic ones for plasma physics, and, among other things, they play an important role in understanding the interaction of high-temperature plasmas with cold gases [15] or the interactions at the edge plasmas in Tokamaks [16]. It is important to emphasize that the features mentioned above in the spectral profiles can be utilized as diagnostics. The whole study is complementary to the analysis of the coupling between charge-exchange processes dynamics and ionic populations dynamics in plasmas, the latter study being addressed to line intensities instead of spectral line shapes [16].

The focus of the survey is at the one-electron ionic quasimolecule. The reason is that, in addition to hot plasma and astrophysics applications, the one-electron quasi-molecule is the unique example of quasi-molecules for which energy terms and dipole moments can be calculated straightforwardly, with any desired precision excluding uncertainties in the input data (those uncertainties on terms and dipole moments are quite typical in the calculations of profiles produced by neutrals collisions.).

\section{Energy Terms and Dipole Optical Transition Moments for the Molecule $Z_{1} e Z_{2}$}

This part is devoted to the calculation of the energy terms and the dipole transition matrix elements and related optical values, for multicharged one electron diatomic quasi-molecules with unequal nuclear charges $Z_{1}$ and $Z_{2}$ ( $Z_{1} e Z_{2}$ notation for the quasi-molecule). The first wave of interest for such molecules was initiated by spectroscopic observations attributed to inner-shell excitations in ionatom collisions [17, 18]. Another wave came from the hot dense plasma physics community. The excited state orbital of mononuclear ion bound electrons may be strongly disturbed by the field of the nearest-neighbor ion. Within these conditions, transitions in quasi-molecules are relevant for the interpretation of experimental results $[10-14,19,20]$.

At first the exchange interaction in the quasi-molecule and its influence on the spectral profiles was considered with the rough approximations of exponential interaction energies and constant dipole matrix elements. These approximations are valid for allowed transitions produced by quasimolecular transitions in the region of large internuclear distances. The approach is unsuitable for forbidden transitions characterized by a strong dependence of the dipole matrix elements on the internuclear distance. The need of accurate values of dipole moments over a wide range of internuclear distances rather than restricted to large ones only is then obvious.

At this step it is important to notice that the dipole matrix elements for the one-electron heterodiatomic quasimolecules $\left(Z_{1} \neq Z_{2}\right)$, in the frame of nonrelativistic approach, had not been calculated before the work done by Devdariani et al. [21]; only the quasi-molecular energy terms had been reviewed in details by Komarov et al. [22].
In the present section we first scale the radiative characteristics of the one-electron heterodiatomic quasi-molecules, then; after summarizing the method of calculation of the energy terms and the dipole moments matrix element, we provide some results and applications to spectroscopy.

2.1. Scaling the Radiative Characteristics. We derive the formulae which relate the radiative characteristics of the oneelectron diatomic quasi-molecule with nuclei charges $Z_{1}$ and $Z_{2}\left(Z_{1} \geq Z_{2}\right)$ to the same characteristics of the one-electron quasi-molecule with nuclei charges $Z_{1}^{\prime}=Z_{1} / Z_{2}$ and $Z_{2}^{\prime}=1$.

For an electron being in the field of two fixed nuclei $Z_{1}$ and $Z_{2}$ separated at the distance $R$, the matrix element of the electric dipole moment operator between states specified by quantum numbers $i, m_{i}$ and $j, m_{j}$ is given by the following formula:

$$
\vec{d}_{i m_{i}, j m_{j}}\left(Z_{1}, Z_{2}, R\right)=\int \psi_{i m_{i}}^{*}(\vec{r}, R) \vec{r} \psi_{j m_{j}}(\vec{r}, R) \overrightarrow{d r}
$$

In (1) $m_{i}$ and $m_{j}$ stand for the magnetic quantum numbers, which determine the projection of the orbital momentum of the electron on the internuclear axis $\vec{R}, \vec{r}$ denotes the position vector of the electron (with origin at the middle of the internuclear axis), and $\psi_{i m_{i}}$ and $\psi_{j m_{j}}$ are the twoCoulomb-centre wave-functions. These eigen-functions are solutions of the Shrödinger equation for the energy terms $\varepsilon_{i\left|m_{i}\right|}(R)$ respectively.

The oscillator strength $f_{i j}\left(Z_{1}, Z_{2}, R\right)$ corresponding to the electric dipole transition $i \rightarrow j$ is determined by means of the matrix element (1), that is,

$$
f_{i j}\left(Z_{1}, Z_{2}, R\right)=-\frac{2}{3} \frac{\omega_{i j}(R)}{g_{i}} \sum_{m_{i}, m_{j}}\left|\vec{d}_{i m_{i}, j m_{j}}\left(Z_{1}, Z_{2}, R\right)\right|^{2},
$$

where $\varrho_{i j}(R)=\boldsymbol{\varepsilon}_{i\left|m_{i}\right|}(R)-\boldsymbol{\varepsilon}_{j\left|m_{j}\right|}(R)$ is the difference between the energy terms, and $g_{i}$ is the degree of degeneracy or statistic weight of the initial level $i\left(g_{i}=1\right.$ for states with $m_{i}=0$ and $g_{i}=2$ for states with $m_{i} \neq 0$ ).

Finally for spontaneous emission by a quasi-molecule $Z_{1} e Z_{2}$ from the state $i$ to $j$, the probability per time unit $A_{i j}\left(Z_{1}, Z_{2}, R\right)$ can be expressed in terms of the oscillator strength of the corresponding transition [23]:

$$
A_{i j}\left(Z_{1}, Z_{2}, R\right)=2 \alpha^{3} \omega_{i j}^{2}(R)\left|f_{i j}\left(Z_{1}, Z_{2}, R\right)\right|,
$$

where $\alpha$ is the fine structure constant.

Let us perform the following scale transformations:

$$
\vec{r}=\frac{\vec{\rho}}{Z_{2}}, \quad \vec{R}=\frac{\vec{L}}{Z_{2}}, \quad Z_{1}^{\prime}=\frac{Z_{1}}{Z_{2}}, \quad Z_{2}^{\prime}=1 .
$$

It can be shown that the normalized eigen-functions and the eigen-values of the two-center Shrödinger equation verify the following relations [24]:

$$
\begin{gathered}
\psi_{i m_{i}}(\vec{r}, R)=Z_{2}^{3 / 2} \varphi_{i m_{i}}\left(Z_{2} \vec{r}, Z_{2} R\right), \\
\boldsymbol{\varepsilon}_{i\left|m_{i}\right|}(R)=Z_{2}^{2} \boldsymbol{\varepsilon}_{i\left|m_{i}\right|}\left(Z_{2} R\right) .
\end{gathered}
$$




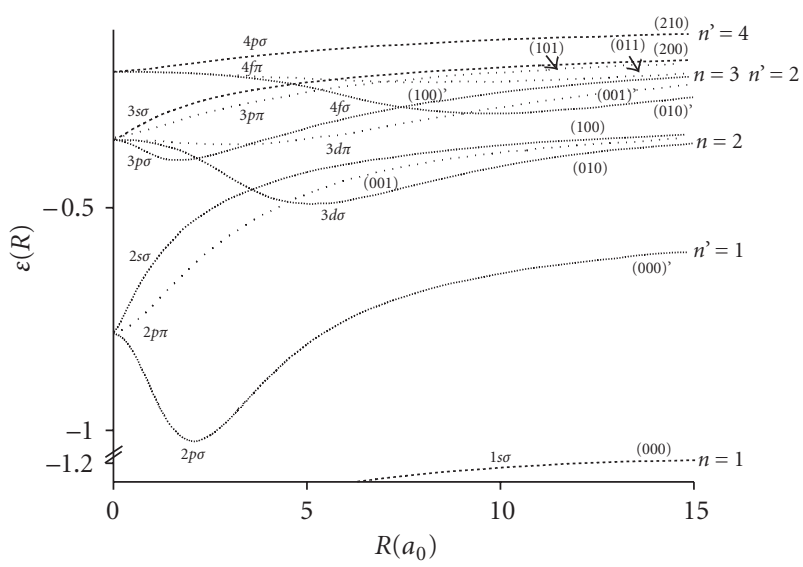

Figure 1: Molecular orbital correlation diagram for the quasimolecule $Z_{1} e Z_{2}$ with $Z_{1}=1.5$ and $Z_{2}=1$. For the notation of the molecular orbitals, we use the united ion designation in the left part of the figure and the parabolic quantum numbers in brackets in the right part of the figure. $n$ stands for the main quantum number in the separated ions limit, and the primed numbers are for the ion with $Z_{1}=1$.

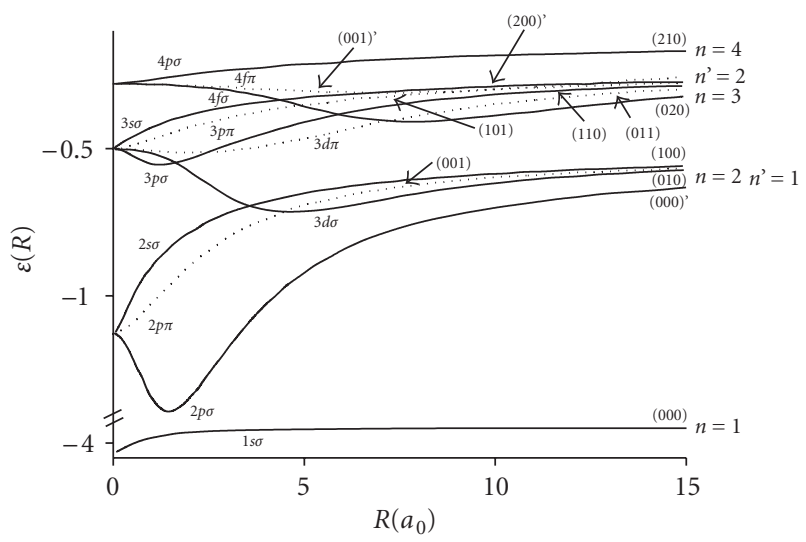

Figure 2: Molecular orbital correlation diagram for the quasimolecule $Z_{1} e Z_{2}$ with $Z_{1}=2.0$ and $Z_{2}=1$. The notations are similar to the ones on Figure 1 .

As a consequence this gives the scaling transformations for the radiative characteristics of the quasi-molecule $Z_{1}^{\prime} e Z_{2}^{\prime}$ experimenting the electric dipole transition $i \rightarrow j$.

$$
\begin{gathered}
\vec{d}_{i m_{i}, j m_{j}}\left(Z_{1}, Z_{2}, R\right)=\frac{1}{Z_{2}} \vec{d}_{i m_{i}, j m_{j}}\left(Z_{1}^{\prime}, Z_{2}^{\prime}, L\right), \\
f_{i j}\left(Z_{1}, Z_{2}, R\right)=f_{i j}\left(Z_{1}^{\prime}, Z_{2}^{\prime}, L\right), \\
A_{i j}\left(Z_{1}, Z_{2}, R\right)=Z_{2}^{4} A_{i j}\left(Z_{1}^{\prime}, Z_{2}^{\prime}, L\right) .
\end{gathered}
$$

In the particular case of homonuclear quasi-molecules $\left(Z_{1}=\right.$ $Z_{2}=Z$ ), the radiative characteristics reduce to the ones relevant to the hydrogen molecular ion $H_{2}^{+}$.

The formulae obtained above reduce the calculation of the radiative characteristics of the one-electron quasimolecules with the nuclei charges $k Z_{1}$ and $k Z_{2}(k=$ $1,2,3, \ldots)$ to the calculation of the same characteristics for a single quasi-molecule with the nuclei charges $Z_{1}^{\prime}=Z_{1} / Z_{2}$ and $Z_{2}^{\prime}=1$. We emphasize that the calculation of the radiative characteristics for the quasi-molecules with smaller nucleus charges is timesaving and faced with smaller computational difficulties.

2.2. The Molecular Orbital (MO) Energy Diagrams and the Dipole Moment Matrix Elements [21]. It is well known that the Schrödinger equation of the two-coulomb-centre system is separable by using the prolate spheroïdal coordinate system $(\xi, \eta, \varphi)$ related to the coordinates $x, y, z$ of the electron position vector through

$$
\begin{gathered}
x=\frac{R}{2} \sqrt{\left(\xi^{2}-1\right)\left(1-\eta^{2}\right)} \cos \varphi, \\
y=\frac{R}{2} \sqrt{\left(\xi^{2}-1\right)\left(1-\eta^{2}\right)} \sin \varphi, \\
z=\frac{R}{2} \xi \eta .
\end{gathered}
$$

The solution of this equation can then be presented as the following product:

$$
\begin{gathered}
\psi_{i m_{i}}(\vec{r}, R)=\frac{1}{\sqrt{2 \pi}} X_{i\left|m_{i}\right|}(\xi, R) Y_{i\left|m_{i}\right|}(\eta, R) e^{i m_{i} \varphi}, \\
1 \leq \xi<\infty,-1 \leq \eta \leq 1,0 \leq \varphi<2 \pi .
\end{gathered}
$$

Here $X_{i\left|m_{i}\right|}(\xi, R)$ and $Y_{i\left|m_{i}\right|}(\eta, R)$ stand for the normalized two-Coulomb-centre quasi-radial and quasi-angular wave functions. After an integration over $\varphi$, the dipole matrix elements, depending on the wave-functions $\psi_{i m_{i}}$ and $\psi_{j m_{j}}$, can be expressed through those forms:

$$
\begin{aligned}
\left(d_{x}\right)_{i m_{i}, j m_{j}}= & \pm i\left(d_{y}\right)_{i m_{i}, j m_{j}} \\
=\frac{R^{4}}{32}\left[\int_{1}^{\infty} X_{i\left|m_{i}\right|} X_{j \mid m_{j}} \mid \sqrt{\xi^{2}-1} \xi^{2} \delta \xi\right. & \\
& \quad \int_{-1}^{1} Y_{i\left|m_{i}\right|} Y_{j\left|m_{j}\right|} \sqrt{1-\eta^{2}} \delta \eta \\
& \quad \int_{1}^{\infty} X_{i\left|m_{i}\right|} X_{j\left|m_{j}\right|} \sqrt{\xi^{2}-1} \delta \xi \\
& \left.\quad \int_{-1}^{1} Y_{i\left|m_{i}\right|} Y_{j \mid m_{j}} \mid \sqrt{1-\eta^{2}} \eta^{2} \delta \eta\right] \\
\left(d_{z}\right)_{i m_{i}, j m_{j}}=\frac{R^{4}}{16}\left[\int_{1}^{\infty} X_{i\left|m_{i}\right|} X_{j\left|m_{j}\right|} \xi \xi^{3} d \xi \int_{-1}^{1} Y_{i\left|m_{i}\right|} Y_{j\left|m_{j}\right| \eta d \eta}\right. & \left.\quad \int_{1}^{\infty} X_{i\left|m_{i}\right|}\left|X_{j\left|m_{j}\right|} \xi d \xi \int_{-1}^{1} Y_{i\left|m_{i}\right|}\right| Y_{j\left|m_{j}\right|} \eta^{3} d \eta\right] .
\end{aligned}
$$


TABle 1: Dipole moment matrix elements for transitions $2 p \sigma \rightarrow 1 s \sigma$ and $3 d \sigma \rightarrow 1 s \sigma$ in relation to the distance $R$ between ions $Z_{1}=$ $1.5,2.0,2.5,3.0$ and $Z_{2}=1$, all values in atomic units.

\begin{tabular}{|c|c|c|c|c|c|c|c|c|}
\hline \multirow{2}{*}{$R$} & \multicolumn{4}{|c|}{$2 p \sigma \rightarrow 1 s \sigma$} & \multicolumn{4}{|c|}{$3 d \sigma \rightarrow 1 s \sigma$} \\
\hline & $Z_{1}=1.5$ & $Z_{1}=2.0$ & $Z_{1}=2.5$ & $Z_{1}=3.0$ & $Z_{1}=1.5$ & $Z_{1}=2.0$ & $Z_{1}=2.5$ & $Z_{1}=3.0$ \\
\hline 0.25 & -0.33803 & -0.28907 & -0.25263 & -0.22412 & -0.00174 & -0.00303 & -0.00404 & -0.00485 \\
\hline 0.50 & -0.41545 & -0.36026 & -0.31328 & -0.27264 & -0.00488 & -0.00885 & -0.01202 & -0.01446 \\
\hline 0.75 & -0.50288 & -0.42748 & -0.35600 & -0.29371 & -0.01044 & -0.01920 & -0.02566 & -0.02989 \\
\hline 1.00 & -0.58488 & -0.47277 & -0.36814 & -0.28570 & -0.01962 & -0.03555 & -0.04541 & -0.05021 \\
\hline 1.50 & -0.70306 & -0.48210 & -0.32696 & -0.23558 & -0.05478 & -0.08810 & -0.09784 & -0.09613 \\
\hline 2.00 & -0.73859 & -0.41656 & -0.26382 & -0.19231 & -0.12002 & -0.15697 & -014965 & -0.13021 \\
\hline 2.50 & -0.68315 & -0.33383 & -0.21462 & -0.16807 & -0.20965 & -0.21854 & -0.18207 & -0.14208 \\
\hline 3.00 & -0.56969 & -0.26313 & -0.18281 & -0.15913 & -0.29820 & -0.25760 & -0.19418 & -0.13747 \\
\hline 3.50 & -0.44511 & -0.20898 & -0.16470 & -0.16077 & -0.36338 & -0.27608 & -0.19380 & -0.12248 \\
\hline 4.00 & -0.33529 & -0.16811 & -0.15710 & -0.16779 & -0.40070 & -0.28197 & -0.18663 & -0.10089 \\
\hline 4.50 & -0.24687 & -0.13647 & -0.15830 & -0.17481 & -0.41596 & -0.28172 & -0.17467 & -0.07747 \\
\hline 5.00 & -0.17850 & -0.11095 & -0.16738 & -0.17913 & -0.41672 & -0.27920 & -0.15713 & -0.05717 \\
\hline 6.00 & -0.08884 & -0.07110 & -0.19803 & -0.18181 & -0.39782 & -0.27401 & -0.10127 & -0.03076 \\
\hline 7.00 & -0.04175 & -0.04202 & -0.21427 & -0.18187 & -0.37434 & -0.27132 & -0.04739 & -0.01693 \\
\hline 8.00 & -0.01880 & -0.02280 & -0.21733 & -0.18147 & -0.35788 & -0.27062 & -0.02052 & -0.00928 \\
\hline 9.00 & -0.00821 & -0.01163 & -0.21755 & -0.18103 & -0.35023 & -0.27079 & -0.00893 & -0.00494 \\
\hline 10.0 & -0.00352 & -0.00570 & -0.21727 & -0.18062 & -0.34912 & -0.27106 & -0.00390 & -0.00255 \\
\hline 12.0 & -0.00062 & -0.00127 & -0.21655 & -0.17992 & -0.35424 & -0.27107 & -0.00074 & -0.00063 \\
\hline 14.0 & -0.00010 & -0.00026 & -0.21591 & -0.17938 & -0.35822 & -0.27060 & -0.00014 & -0.00014 \\
\hline 16.0 & -0.00002 & -0.00005 & -0.21537 & -0.17896 & -0.35959 & -0.27004 & -0.00002 & -0.00003 \\
\hline 18.0 & 0.00000 & -0.00001 & -0.21492 & -0.17862 & -0.35979 & -0.26951 & 0.00000 & -0.00001 \\
\hline 20.0 & 0.00000 & 0.00000 & -0.21455 & -0.17834 & -0.35957 & -0.26904 & 0.00000 & 0.00000 \\
\hline 22.0 & 0.00000 & 0.00000 & -0.21423 & -0.17810 & -0.35922 & -0.26862 & 0.00000 & 0.00000 \\
\hline 24.0 & 0.00000 & 0.00000 & -0.21397 & -0.17790 & -0.35883 & -0.26825 & 0.00000 & 0.00000 \\
\hline 26.0 & 0.00000 & 0.00000 & -0.21373 & -0.17773 & -0.35844 & -0.26793 & 0.00000 & 0.00000 \\
\hline 28.0 & 0.00000 & 0.00000 & -0.21353 & -0.17759 & -0.35807 & -0.26764 & 0.00000 & 0.00000 \\
\hline 30.0 & 0.00000 & 0.00000 & -0.21336 & -0.17746 & -0.35773 & -0.26739 & 0.00000 & 0.00000 \\
\hline$\infty$ & 0.00000 & 0.00000 & -0.21070 & -0.17558 & -0.35117 & -0.26337 & 0.00000 & 0.00000 \\
\hline
\end{tabular}

They satisfy the selection rules for the magnetic quantum number in the case of dipole transitions, that is, $m_{j}=m_{i} \mp 1$ in (9) for the radiation polarized in the plane perpendicular to $\vec{R}$ ( $\sigma-\pi$ transitions $)$ and $m_{j}=m_{i}$ in (10) for the radiation polarized along the internuclear axis $\vec{R}(\sigma-\sigma, \pi-$ $\pi$ transitions).

The wave functions have been expanded in various forms involving coefficients determined by recurrence relations [21] and yielding the relative accuracy of order $10^{-12}$ for the energy terms and the accuracy of order $10^{-8}-10^{-10}$ for the corresponding quasiradial and quasiangular wave functions. Thus, the accuracy of the calculated matrix elements can be estimated at $10^{-8}$ [21].

The dipole matrix elements (and all energy terms involved) have been calculated for all transitions between the states with the principal quantum number in the united ion limit $n_{u}=1,2,3$ and for four transitions involving $n_{u}=4$. The calculations have been carried out for the quasimolecules with the nucleus charges $Z_{1}=1.5,2,2.5,3$ and $Z_{2}=1$. Some results are given in Tables 1 and 2 for the dipole matrix elements and in Figures 1, 2, and 3 for the energy terms (MO) correlation diagrams. The choice of these results gives examples relevant to the discussion of the most important new features.

In this paper we will not be concerned with the existence of extrema in the energy terms; this feature has been previously used for high-density plasma spectroscopy [10$14,19,20]$.

The most prominent other new features that can be deduced from the data are as follows: (i) the matrix elements strongly depend on the internuclear distance $R$; (ii) some matrix elements of the quasi-molecules, with different $Z_{1}$ at large $R$, exchange their limiting values; (iii) the limiting values of some matrix elements, at large $R$, tend in pairs to the same values or to values of equal modulus and opposite signs, and some of them are equal to zero; (iv) some of the matrix elements have zeros at intermediate $R$.

Below, some features are discussed qualitatively in more detail through examples.

2.2.1. The Limiting Values. A novel feature for the heteronuclear quasi-molecules is that the correlation diagram, 
TABle 2: Dipole moment matrix elements for transitions $3 p \sigma \rightarrow 1 s \sigma$ and $4 p \sigma \rightarrow 1 s \sigma$ in relation to the distance $R$ between ions $Z_{1}=$ $1.5,2.0,2.5,3.0$ and $Z_{2}=1$, all values in atomic units.

\begin{tabular}{|c|c|c|c|c|c|c|c|c|}
\hline \multirow{2}{*}{$R$} & \multicolumn{4}{|c|}{$3 p \sigma \rightarrow 1 s \sigma$} & \multicolumn{4}{|c|}{$4 p \sigma \rightarrow 1 s \sigma$} \\
\hline & $Z_{1}=1.5$ & $Z_{1}=2.0$ & $Z_{1}=2.5$ & $Z_{1}=3.0$ & $Z_{1}=1.5$ & $Z_{1}=2.0$ & $Z_{1}=2.5$ & $Z_{1}=3.0$ \\
\hline 0.25 & -0.12796 & -0.10778 & -0.09289 & -0.08137 & -0.07421 & -0.06223 & -0.05341 & -0.04662 \\
\hline 0.50 & -0.13743 & -0.11347 & -0.09481 & -0.08004 & -0.07673 & -0.06251 & -0.05167 & -0.04327 \\
\hline 0.75 & -0.13436 & -0.10445 & -0.08230 & -0.06729 & -0.07096 & -0.05396 & -0.04225 & -0.03417 \\
\hline 1.00 & -0.11617 & -0.08495 & -0.06615 & -0.05399 & -0.05717 & -0.04080 & -0.03165 & -0.02597 \\
\hline 1.50 & -0.06109 & -0.04839 & -0.04293 & -0.03791 & -0.02412 & -0.01944 & -0.01814 & -0.01653 \\
\hline 2.00 & -0.01617 & -0.02939 & -0.03216 & -0.03033 & -0.00027 & -0.00881 & -0.01182 & -0.01179 \\
\hline 2.50 & 0.00886 & -0.02113 & -0.02680 & -0.02630 & 0.01284 & -0.00385 & -0.00833 & -0.00881 \\
\hline 3.00 & 0.01882 & -0.01735 & -0.02389 & -0.02393 & 0.01881 & -0.00122 & -0.00606 & -0.00665 \\
\hline 3.50 & 0.02062 & -0.01549 & -0.02229 & -0.02235 & 0.02099 & 0.00036 & -0.00442 & -0.00494 \\
\hline 4.00 & 0.01898 & -0.01459 & -0.02142 & -0.02111 & 0.02138 & 0.00139 & -0.00313 & -0.00352 \\
\hline 4.50 & 0.01626 & -0.01426 & -0.02095 & -0.02001 & 0.02096 & 0.00211 & -0.00207 & -0.00231 \\
\hline 5.00 & 0.01345 & -0.01431 & -0.02062 & -0.01897 & 0.02016 & 0.00264 & -0.00114 & -0.00126 \\
\hline 6.00 & 0.00871 & -0.01520 & -0.01989 & -0.01705 & 0.01817 & 0.00340 & 0.00044 & 0.00047 \\
\hline 7.00 & 0.00537 & -0.01666 & -0.01885 & -0.01537 & 0.01619 & 0.00403 & 0.00177 & 0.00182 \\
\hline 8.00 & 0.00313 & -0.01808 & -0.01766 & -0.01394 & 0.01447 & 0.00465 & 0.00290 & 0.00290 \\
\hline 9.00 & 0.00169 & -0.01888 & -0.01646 & -0.01273 & 0.01306 & 0.00529 & 0.00386 & 0.00378 \\
\hline 10.0 & 0.00080 & -0.01894 & -0.01536 & -0.01171 & 0.01197 & 0.00593 & 0.00469 & 0.00452 \\
\hline 12.0 & 0.00004 & -0.01787 & -0.01346 & -0.01007 & 0.01060 & 0.00714 & 0.00604 & 0.00568 \\
\hline 14.0 & -0.00010 & -0.01641 & -0.01194 & -0.00882 & 0.01021 & 0.00821 & 0.00709 & 0.00656 \\
\hline 16.0 & -0.00008 & -0.01504 & -0.01072 & -0.00785 & 0.01082 & 0.00913 & 0.00793 & 0.00725 \\
\hline 18.0 & -0.00005 & -0.01383 & -0.00971 & -0.00707 & 0.01228 & 0.00993 & 0.00862 & 0.00781 \\
\hline 20.0 & -0.00002 & -0.01277 & -0.00887 & -0.00643 & 0.01367 & 0.01061 & 0.00919 & 0.00826 \\
\hline 22.0 & -0.00001 & -0.01186 & -0.00817 & -0.00589 & 0.01448 & 0.01120 & 0.00968 & 0.00865 \\
\hline 24.0 & -0.00001 & -0.01106 & -0.00756 & -0.00544 & 0.01504 & 0.01172 & 0.01010 & 0.00897 \\
\hline 26.0 & 0.00000 & -0.01036 & -0.00704 & -0.00505 & 0.01552 & 0.01219 & 0.01046 & 0.00926 \\
\hline 28.0 & 0.00000 & -0.00973 & -0.00659 & -0.00471 & 0.01596 & 0.01260 & 0.01078 & 0.00950 \\
\hline 30.0 & 0.00000 & -0.00918 & -0.00619 & -0.00442 & 0.01637 & 0.01296 & 0.01107 & 0.00972 \\
\hline$\infty$ & 0.00000 & 0.00000 & 0.00000 & 0.00000 & 0.02621 & 0.01966 & 0.01573 & 0.01311 \\
\hline
\end{tabular}

which relates the orbitals of the isolated and united ions, now depends on $Z_{1}$. More precisely, the rearrangement for the dipole matrix elements of the Molecular Orbital (MO) correlation diagrams results in the dependence of the limiting values on $Z_{1}$, apart from the trivial scaling, and in the exchange of the limiting values. To exemplify the dependence of the limiting values on $Z_{1}$ we take the particular case of the matrix element $\left\langle 010\left|d_{z}\right| 000\right\rangle=$ $-128 / 243 Z_{1}$ for the hydrogen-like ion (for large $R$ the matrix elements are in parabolic coordinates). According to Figures 1-3 and Table 1 this matrix element is the limiting value for the transition between the $3 d \sigma$ and $1 s \sigma$ states in the case of $Z_{1}=1.5,2$; but in the case $Z_{1}=2.5,3$, this matrix element is the limiting value for the transition between the $2 p \sigma$ and $1 s \sigma$ states. Also Figures $1-3$ shows that the $2 s \sigma, 2 p \pi, 3 s \sigma, 3 p \pi$ $\mathrm{MO}$ energy diagrams do not depend on $Z_{1}$.

2.2.2. The Roots of the Optical Transition Dipole Moments. When the dipole matrix element between two states of an atom or an ion is equal to zero, the transition is forbidden. Nevertheless the interaction between particles during the collisions will allow the dipole matrix elements to become functions of internuclear distance and the optical transitions to be allowed. Such optical transitions can be identified as true quasi-molecular ones, and up to now some of these transitions produced in atomic and ionic collisions have been studied [26-28].

The existence of zeros in the dipole transition matrix elements had already been pointed out for symmetrical quasi-molecules $[31,32]$. In the hetero-nucleus case, the parallel transitions, having zeros in the symmetrical case, have zeros for all $Z_{1}$ values considered here, namely, 4po$1 s \sigma$; see Table 2 . The matrix element for the transition $3 p \sigma-$ $1 s \sigma$ has two zeros but only for $Z_{1}=1.5$ and has no zero otherwise; see Table 2.

In the hetero-nuclear quasi-molecules there is an additional possibility for asymptotically forbidden-transitions. When at $R \rightarrow \infty$ the upper and lower states belong to different ions, the corresponding wave functions do not overlap, and therefore, the matrix element is equal to zero. At smaller $R$, the interaction between two different ions will allow optical transitions corresponding to the transition 
of one electron from one ion to the other. Such optical transitions involve a nonresonant charge-exchange process and they correspond to asymptotically forbidden transitions. As examples the transitions $2 p \sigma-1 s \sigma$ for $Z_{1}=1.5,2$ and $3 d \sigma-1 s \sigma$ for $Z_{1}=2.5,3$ accompanying the charge-exchange between the lowest ion states with parabolic sets $(000)$ and $(000)^{\prime}$ correspond to such asymptotically forbiddentransitions (see Table 1).

The results of these calculations put forward two problems: what is the reason for the roots and how can we describe the spectral features produced?

\section{Spectral Features Due to Intersection of Energy Terms}

In this part we will show that the intersection of terms not only leads to the formation of extrema in energy terms but can also be responsible for the roots of dipole moments. As for the spectral features, an extremum in an energy term will then not necessarily produce a satellite. Of particular interest is the role of dipole transition moments [21,33], which can lead, in the case of zeros, to the formation of dips in the far wing spectral profiles [21]. This phenomenon has been discussed in two limiting cases, adiabatic and diabatic [34].

3.1. The Two-State Model for the Dipole Moments. The prediction of roots and their positions can be made within the two-state model.

Let us next consider the two-state adiabatic and diabatic wave functions $\left|\Psi_{I, I I}\right\rangle$ and $\left|\varphi_{1,2}\right\rangle$, for the quasi-molecule, respectively. We now follow the Nikitin model [25] that is appropriate to have an idea of all situations and can reveal the main conclusions. The model gives for the diabatic matrix elements $V_{i k}=\left\langle\varphi_{i}|V| \varphi_{k}\right\rangle$ of the interaction between the two ions:

$$
\begin{aligned}
V_{i k}= & \delta_{i k}\left[V(x)+\frac{\Delta \varepsilon}{2}\left(1-e^{-x} \cos 2 \Theta_{0}\right)(-1)^{1+i}\right] \\
& +\left(1-\delta_{i k}\right) \frac{\Delta \varepsilon}{2} e^{-x} \sin 2 \Theta_{0} .
\end{aligned}
$$

The variable $x$ is a dimensionless distance $(x=0$ at the center of the considered nonadiabatic region). The parameter $\Theta_{0}$ characterizes two alternative evolutions of the energy terms in the considered transition zone, namely, intersecting, $0 \leq$ $\Theta_{0} \leq \pi / 4$, (Figure $4(\mathrm{a})$ ) or nonintersecting, $\pi / 4 \leq \Theta_{0} \leq \pi / 2$, (Figure $4(\mathrm{~b})$ ) diabatic terms. $\Delta \varepsilon$ is the splitting between the terms at large distances.

The same model leads to the following adiabatic dipole moments:

$$
D=\sqrt{d_{1}^{2}+d_{2}^{2}}\left|\begin{array}{c}
\cos \left(\Theta-\Theta_{d}\right) \\
-\sin \left(\Theta-\Theta_{d}\right)
\end{array}\right| .
$$

In this formula, the dipole moments for optical transitions towards the ground state $\left|\varphi_{0}\right\rangle$ are defined on the two wave function basis (adiabatic and diabatic) as $D_{I, I I}=$ $\left\langle\Psi_{I, I I}|d| \varphi_{0}\right\rangle \cdot d_{1,2}=\left\langle\varphi_{I, I I}|d| \varphi_{0}\right\rangle$, and tg $\Theta_{d}=d_{2} / d_{1}\left(\Theta_{d}\right.$ varying from 0 to $\pi / 2$ if $d_{1} d_{2} \geq 0$ ).

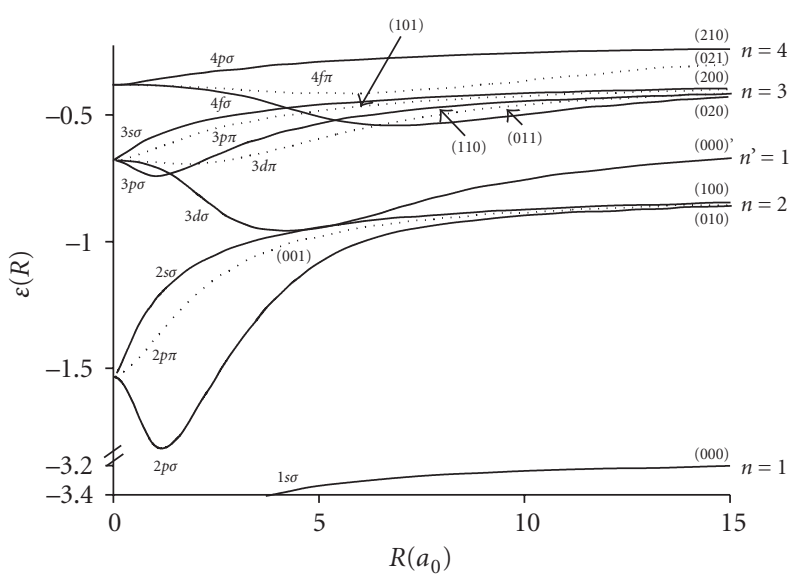

FIgURE 3: Molecular orbital correlation diagram for the quasimolecule $Z_{1} e Z_{2}$ with $Z_{1}=2.5$ and $Z_{2}=1$. The notations are similar to the ones on Figure 1.

The free parameter $\Theta$, varying from $\pi / 2-\Theta_{0}$ to 0 , when $x$ varies from $-\infty$ to $+\infty$, is defined as follows:

$$
\begin{gathered}
\Theta=\operatorname{arctg} \sqrt{\frac{K-\Delta}{K+\Delta}} \text { with } \Delta=V_{11}-V_{22}, \\
K=\sqrt{\Delta^{2}+4\left|V_{12}\right|^{2}} .
\end{gathered}
$$

3.2. The Roots of the Dipole Moments and the Spectral Profiles. In this section, we discuss the adiabatic dipole transition moment roots and their manifestations in the spectral profiles (satellites and/or dips). For this purpose, the relationship between $\Theta_{0}$ and $\Theta_{d}$ turns out to be determining (see Figure 5, where all possible cases are summarized). As one can see from (12), one of the two adiabatic dipole transition moment has a root at $\Theta=\Theta_{d}$ and another has a maximum if the upper limiting value $\Theta(x \rightarrow-\infty)=$ $\pi / 2-\Theta_{0} \geq \Theta_{d}$ (regions $I I I$ and $I V$ on Figure 5 ). But there are no roots in the opposite case (regions $I$ and $I I$ ). We should stress that counter-intuitively, there is a root in the former case for nonintersecting diabatic terms corresponding to $\Theta_{0} \geq \pi / 4$ (region $I I I$ ) but no root for intersecting diabatic terms corresponding to $\Theta_{0} \leq \pi / 4$ (region $I I$ ). Thus the main conclusion is that the intersection of terms is neither a necessary nor a sufficient condition for the existence of a dipole moment root.

Then we discuss the effect, on the optical spectral profiles, of the dipole transition moment rearrangement in the four regions previously discussed in the space $\Theta_{0}, \Theta_{d}$.

In the region $I$ of Figure 5, the adiabatic potential energy curves (and consequently the transition energies) do not have extrema $\left(\pi / 4<\Theta_{0}<\pi / 2\right.$, Figure $\left.4(\mathrm{~b})\right)$, and the dipole moments do not have any root at any allowed interatomic distances. This case leads to a monotonous dependence of the profiles on frequency.

In the region $I I$, every adiabatic potential energy curve (and consequently the transition energy) has an extremum $\left(\Theta_{0}<\pi / 4\right.$, Figure 4(a)), but the dipole moments have neither roots nor extrema. It is well known that an extremum 


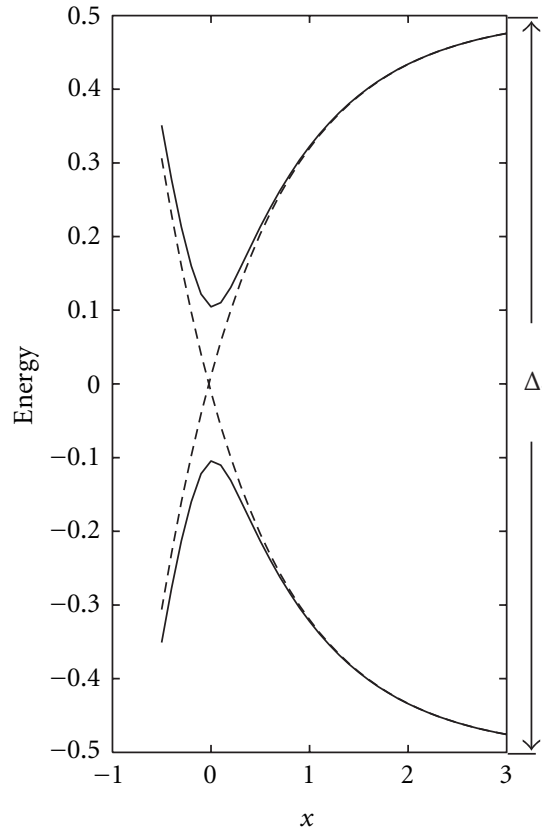

(a) Case (a): intersecting diabatic energy terms

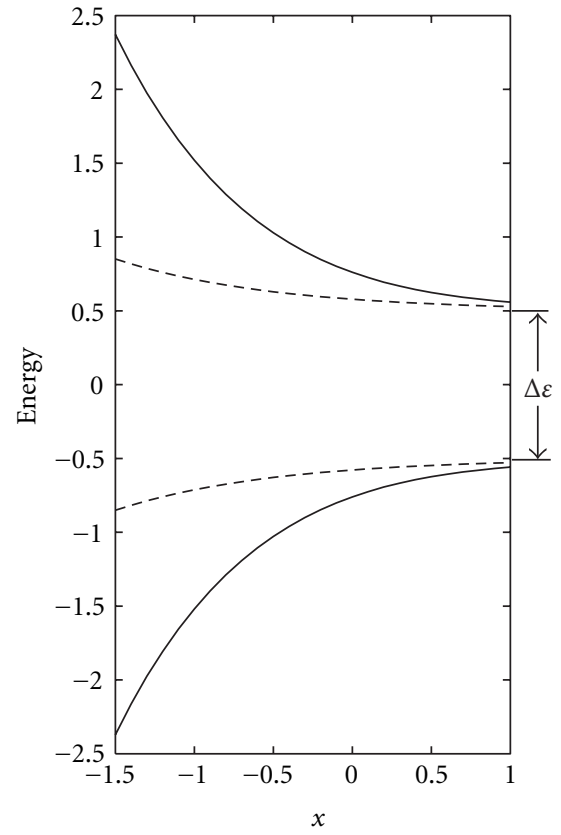

(b) Case (b): nonintersecting diabatic energy terms

FIgURE 4: The two alternative evolutions of the diabatic energy terms $V_{11}$ and $V_{22}$ (the dashed lines) with respect to $x$ (dimensionless distance), within the Nikitin's model [25]. The solid lines stand for the adiabatic energy terms. The energies are in arbitrary unit, and $\Delta \varepsilon$ is the splitting between the terms at large distances. Case (a): intersecting diabatic terms. Case (b): nonintersecting diabatic terms.

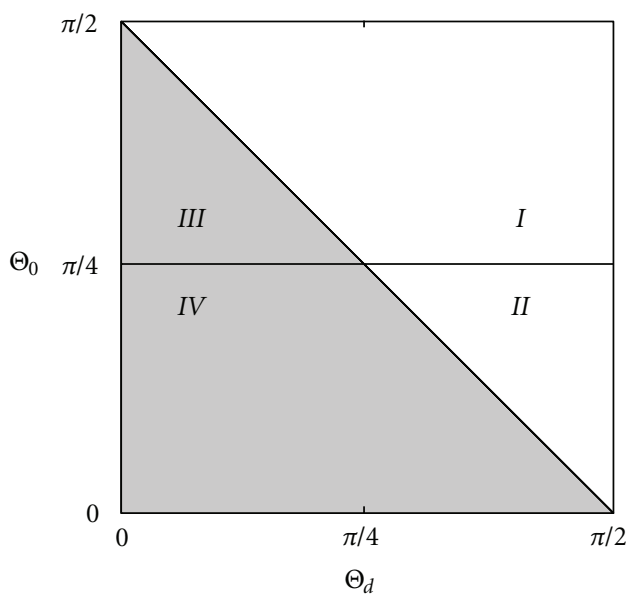

FIGURE 5: The occurrence of the adiabatic dipole transition moment roots depending on the relationship between the parameters $\Theta_{d}, \Theta_{0}$. A root exists at $\Theta_{0}+\Theta_{d} \leq \pi / 2$, that is, in the regions $I I I, I V$. For parameters in the regions $I$, III the diabatic potential energy curves do not cross each other contrary to the regions II and IV. The ordinates $\Theta_{0}=0, \pi / 4$ correspond to Landau-Zener's and Demkov's models, respectively. The abscissas $\Theta_{d}=0, \pi / 2$ correspond to the forbidden dipole transitions, for example, [25].

in the transition energy produces a satellite in the spectral profile.

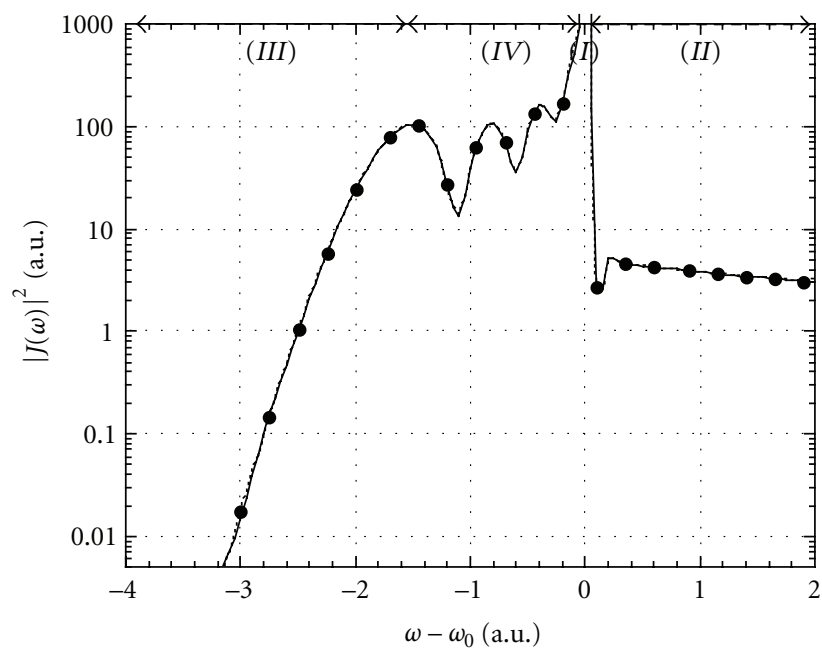

FIGURE 6: Visualization of the four regions in the line shape $|J(\omega)|^{2}$ of the transition $2 p \pi_{u} \rightarrow 1 s \sigma_{g}$ for the molecule $F^{8+}-F^{9+}$, according to (17) (dots), [29]. Results of numerical calculations for $|J(\omega)|^{2}$ are given by (15) and (16) for rectilinear trajectories (solid line). Parameters are $d=2.3 \cdot 10^{-2} Z^{2}, r_{e x}=4.2 / Z, v=0.035$. Iline core; $I I$-blue wing; $I I I$-antistatic region; $I V$-region of quantum oscillations due to the minimum of the potential-difference.

Region III of Figure 5 is completely distinctive from these two previous regions with the simultaneous existence of a 
maximum of the dipole moment for the upper energy state and a root for the lower state at $\Theta_{d}=\operatorname{arctg}\left(d_{2} / d_{1}\right)$, without any extremum in the potential energy (and in the transition energy) curves $\left(\pi / 4<\Theta_{0}<\pi / 2\right.$, Figure $\left.4(\mathrm{~b})\right)$. Clearly the lower state produces a dip in the profile.

Finally, in the region $I V$ there is an extremum in the two adiabatic transition energy terms at $R=R_{m}$ jointly with a maximum for $d_{I}$ (the upper state) and a root for $d_{I I}$ (the lower state) at $R=R_{d}$. In general $R_{m} \neq R_{d}$, and thus the upper state produces two satellites, one connected with the minimum in the adiabatic transition energy curve, and another connected with the maximum in the adiabatic dipole moment. The lower state produces a satellite due to the minimum in the adiabatic transition energy curve and $a$ dip. The spectral features are more clearly pronounced when $R_{m}=R_{d}$; this situation leads to the merging of the two satellites in one (for the upper state case) and to the merging of the dip and the satellite in some structure (for the lower state case).

\section{Quasimolecular Optical Transitions Followed by Charge-Exchange}

4.1. Symmetric Charge-Exchange. We start from the description of radiative transitions in symmetric quasi-molecules. The process is schematically described as follows:

$$
(Z e)^{*}+Z \longrightarrow Z+(Z e)+\hbar \omega
$$

and can be realized by two ways as a radiative transition between one initial quasi-molecular state and two possible final quasi-molecular states. The first way is roughly an optical transition that can be described qualitatively as a transition in an excited ion $(Z e)^{*}$ broadened by collisions with a charged particle $Z$, mainly at large interionic distances. The second way can be described as an optical transition which is followed by a jump of an electron from one charged center to another and takes place mainly at small and moderate interionic distances. It should be stressed that the probability of the reaction (14) in both ways is determined by the probabilities of the optical transitions involved. Of course the final state in a distant ion is the same for both ways, so that the correct description of the reaction (14) must take into account an interference because of the two possible reaction ways.

As it follows from the qualitative discussion, the decisive factor for the reaction (14) is the influence of "one-electron" exchange interaction. Using Ly $\alpha$ as an example it has been shown in $[29,35]$ that exchange interaction produces noticeable structures in the spectral profile of Ly $\alpha$ radiation. The rst indication of the structure can be obtained from the quasistatic theory [36] and the asymptotic theory of the charge exchange [37]. The interaction between ions transforms the ground ionic state into the quasi-molecular states $1 s \sigma_{g}$ and $2 p \sigma_{u}$, the excited $2 s$ state into the quasimolecular states $\sigma_{g, u}$, and the excited $2 p$ state into the $\sigma_{g, u}$ and $\pi_{g, u}$ states. At large interionic distances the splitting of the ground states is proportional to $\exp (-Z r)$, while the splitting of the excited states is proportional to $\exp (-Z r / 2)$.
In addition, the splitting for $\pi$ terms is opposite in sign to that for $\sigma$ terms [37]. Therefore, the potential-difference curve $\Delta U_{+}=U\left(3 d \pi_{g}\right)-U\left(2 p \sigma_{u}\right)$ experiences a maximum and the potential-difference curve $\Delta U_{-}=U\left(2 p \pi_{u}\right)-U\left(1 s \sigma_{g}\right)$ experiences a minimum, As predicted even by the quasistatic theory, these extrema lead to the formation of structures in the spectral wings of perpendicular optical transitions $3 d \pi_{g} \rightarrow 2 p \sigma_{u}, 2 p \pi_{u} \rightarrow 1 s \sigma_{g}$.

The total intensity distribution is shown to be proportional to

$$
|b(\omega, t)|^{2}=\frac{1}{2}\left(\left|J_{+}\right|^{2}+\left|J_{-}\right|^{2}\right)
$$

where $J_{ \pm}$are the amplitudes of the two quasi-molecular radiative transitions discussed above [29], and

$$
J_{ \pm}(\omega, t)=\int_{t_{0}}^{t}\left\langle\omega_{1}|V| \varphi_{1}\right\rangle \exp \left(-i \int_{t_{0}}^{t^{\prime}}\left(\Delta U_{ \pm}-\omega\right) d t^{\prime \prime}\right) d t^{\prime}
$$

and $\Gamma_{1,2}=2 \pi\left|\left\langle\omega_{1,2}|V| \varphi_{1,2}\right\rangle\right|^{2}$ are the radiative width. The reasonable approximation for $\Delta U_{ \pm}$by exponential functions leads to global approximation for spectral line shapes

$$
\begin{aligned}
\frac{J(\omega)}{\left\langle\omega_{1}|V| \varphi_{1}\right\rangle}= & j(\omega) \\
= & \frac{-i}{\alpha}(x)^{-i \Omega} \Gamma(i \Omega) D_{-i \Omega}\left(2 x e^{-3 i \pi / 4}\right) \\
& \times \exp \left(i \alpha \Omega t_{0}+i x^{2}+\frac{\pi \Omega}{4}\right)
\end{aligned}
$$

where $\Omega=(\Delta \omega+i(\Gamma / 2)) / \alpha, \Delta \omega=\omega-\Delta U(r \rightarrow \infty), x=$ $\sqrt{d / \alpha}$.

The formula (17) gives an analytical description in four different regions of the spectral profile: a Lorentzian region situated near the center $\Delta \omega \approx 0$, a blue wing with one Condon point, an antistatic region of classically forbidden transitions with no Condon point, and a region corresponding to transitions near the extrema of the potential-difference curve. Figure 6 gives the example of the Ly $\alpha$ profile produced in $F^{+8}-F^{+9}$ collisions.

The main conclusion of $[29,35]$ is that exchange interaction results in the formation of two satellites perpendicularly polarized. It is interesting to point out that the main idea of the asymptotic approach for the description of the interaction between atomic/ionic particles is to separate the effects of the long-range and the exchange parts [37]. The long-range part of the $Z e-Z$ interaction produces structures that are in principle similar to structures described under the conventional Stark broadening scheme in plasmas, whereas the exchange interaction produces the features discussed above. As an example, the splitting between $3 d \pi_{g}$ and $2 p \pi_{u}$ terms due to exchange interaction is given by $-Z^{4} r^{2} / 4 \exp (-2-Z r / 2)$ according to [22]. The exchange interaction is equal to the first term of the long-range interaction $6 / Z r^{3}$ at $r_{0} \approx 16 / Z$, meaning that the Stark effect dominates at $r>r_{0}$ and the $g$ - $u$ splitting can be neglected. Therefore the structures produced by the long-range part of 


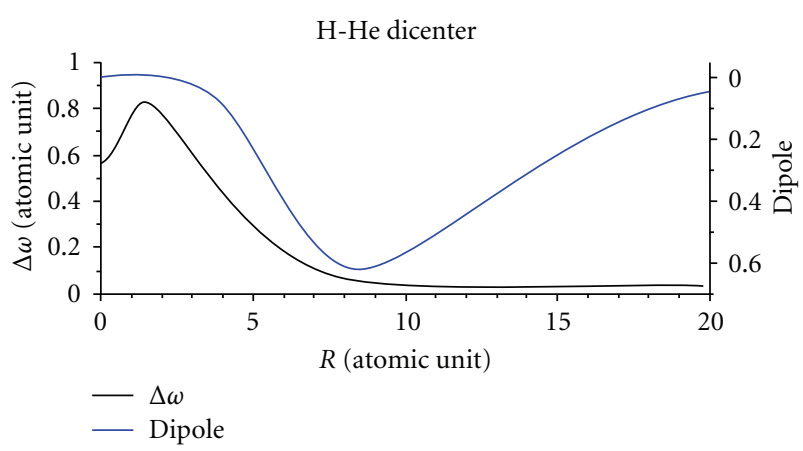

(a)

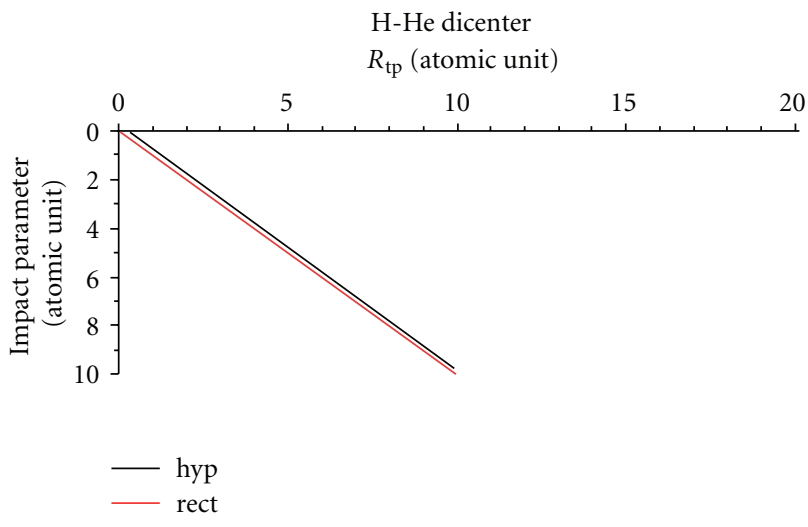

(b)

Figure 7: Dipole transition moment and difference potential $\Delta \omega$ for the reaction (21), (a) The dependence of the turning point position $R_{\mathrm{tp}}$ on the impact parameter (for collision energy la.u.), for rectilinear (rect), and hyperbolic (hyp) trajectories, (b).

the interaction are more sensitive to the plasma surrounding than the ones due to the exchange interaction. In connection with plasma problems, we note that the effect of charge exchange on atomic spectral line shapes in plasmas was analyzed in the frame of the asymptotic theory for exchange interaction of radiating atom with perturbing ion [38].

Equation (17) is applicable to the description of line shapes in the case of any extremum in the potential difference curves characterizing ionic or atomic quasi-molecules. Using this formula in [39] has led to a perfect agreement with experimental data on the spectral profiles produced in $\mathrm{Ca}\left(4^{1} S \rightarrow 3^{1} \mathrm{D}\right)+\mathrm{He}$ forbidden transitions.

4.2. Nonsymmetric Charge-Exchange Involving Accidental Resonance. For nonsymmetrical quasi-molecules, and when the condition

$$
\frac{Z_{1}}{n_{1 f}}=\frac{Z_{2}}{n_{2 f}} \quad \text { or } \frac{Z_{1}}{n_{1 i}}=\frac{Z_{2}}{n_{2 i}}
$$

( $n_{1 i f, 2 i f}$ being the principal quantum numbers of the electron in a corresponding ion) is fulfilled, then the emission of photons can be once more realized via two different optical transitions similar those described above. In ion collision physics, the condition (18) is named "swapping" [17].

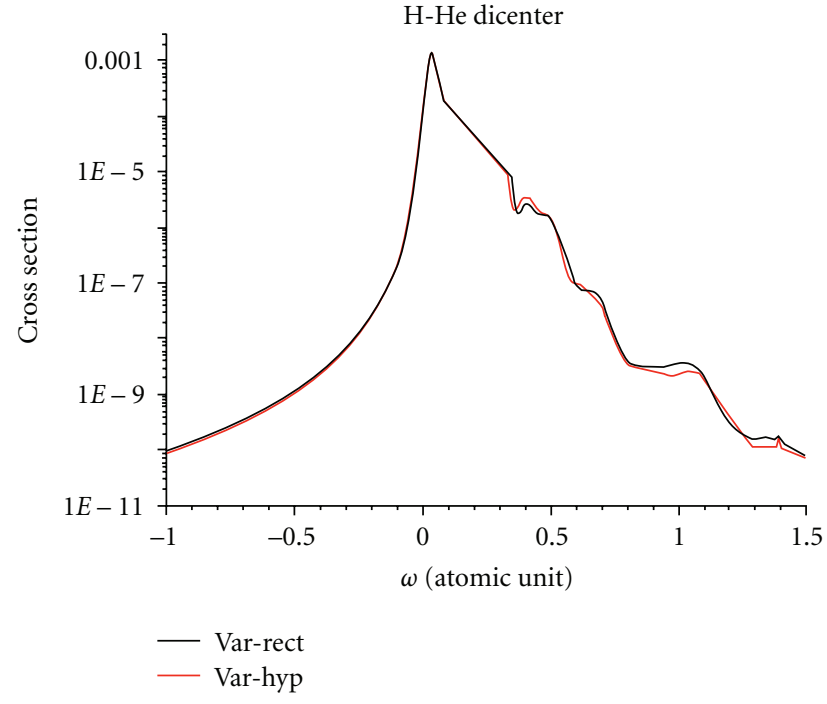

Figure 8: Spectral profile produced in reaction (21) for both rectilinear and hyperbolic trajectories for collision energy 1a.u., [30]. The maximum is shifted about $180 \AA$ from the center of line $H_{\alpha}=1641 \AA$. Some additional structures in the blue wing are due to transitions near the turning points, the extremum of the difference potential and the root of the dipole moment at $R \approx 3$.

The first optical transition is

$$
Z_{1} e\left(n_{1 i}\right)+Z_{2} \longrightarrow Z_{1} e\left(n_{1 f}\right)+Z_{2}+\hbar \omega,
$$

and the second one is an optical transition followed by nonsymmetric charge-exchange

$$
Z_{1} e\left(n_{1 i}\right)+Z_{2} \longrightarrow Z_{1}+Z_{2} e\left(n_{2 f}\right)+\hbar \omega .
$$

A peculiarity of the quasi-molecular optical transition (20) is that its dipole transition moment is equal to zero at large interionic distances. From this point of view, the transition must be attributed to optical forbidden transitions that are mainly concentrated near the position of the dipole moment extremum. Certainly, those two transitions (19) and (20) do not interfere with each other in contrast to the ones discussed previously, so that there will be in general only one satellite produced by the reaction (20) and shifted from the position of an optical transition in a separate ion $Z_{1} e\left(n_{1 i}\right) \rightarrow$ $Z_{1} e\left(n_{1 f}\right)$.

A typical example of the reaction (20) is the following reaction relevant to thermonuclear physics:

$$
H e^{+}(n=3)+H^{+} \longrightarrow H e^{2+}+H(n=1)+\hbar \omega,
$$

producing a blue satellite of the $\mathrm{H \alpha}$ transition in $\mathrm{He}^{+}$; see Figures 7 and 8 for details.

As one can see the transitions near the position of the dipole moment extremum produce a noticeable spectral feature. The reason is clear as the position of the extremum depends neither on the impact parameter, nor on the collision energy; however the half-width of the spectral distribution on Figure 8 depends on collision energy and can be used to determine the temperature of plasma. 


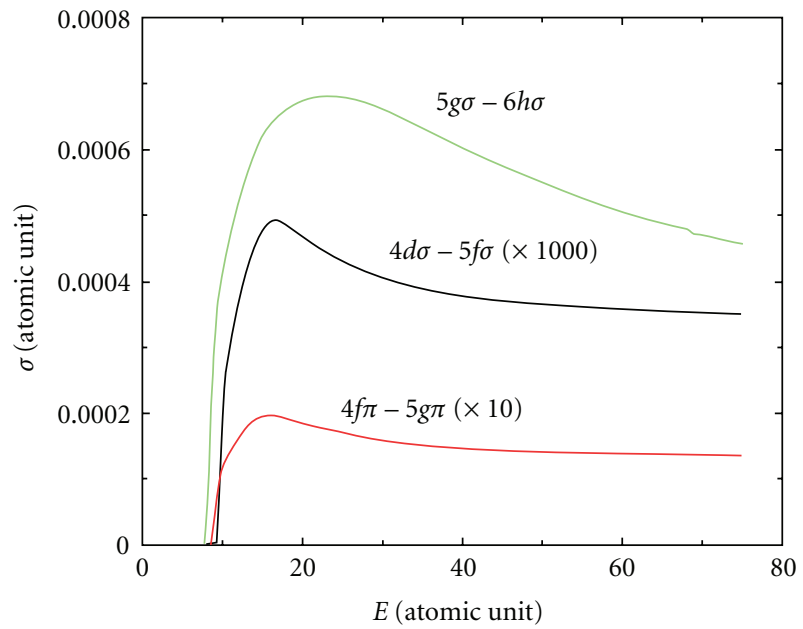

FIGURE 9: Energy evolution of charge exchange $A l^{+12}(n=4)+$ $C^{+6} \rightarrow A l^{+13}+C^{+5}(n=2)$ cross sections for different channels.

4.3. Charge-Exchange in Excited States with Small Energy Defect. Let us suppose that the swapping condition (18) has been disturbed a little, for example,

$$
\frac{Z_{1}}{n_{1 i}} \geq \frac{Z_{2}}{n_{2 i}} .
$$

A representative example of this case is $A l^{+12}(n=4)+C^{+6}$ collisions that have been studied in experiments on hot dense plasmas produced by laser pulses [10-14, 40]. Despite the energy splitting at large distances, two states still exist with asymptotically allowed and forbidden transitions. But the novelty, in comparison with the discussion above, is an avoided crossing between quasi-molecular terms of the two states involved. The energy separation of adiabatic terms $V_{12}$ in the region of avoided crossing is proportional to the exchange interaction at large distances and is rather small thus making easier the diabatic scenario of collisions. In this case (14) is also true, and it predicts the occurrence of "steps" in the dependence of dipole moments on interionic distance, these "steps" occurr in the narrow regions of interionic distances about $\left|\mathrm{V}_{12}\right| / \Delta F, \Delta F$ representing the difference between the forces acting on the two interacting states.

Under (22), an excited state of a separate ion $Z_{1} e\left(n_{i}\right)$ can be depopulated by two qualitatively different ways, namely by optical transitions and by charge-exchange between two excited states. In the diabatic basis, the intersection of interacting diabatic states, one of them is populated, leads to a sudden population of the other state in the narrow region of intersection. The population of this kind can be regarded as a shake and thus produces structures in the spectral profiles similar to the ones observed in Fresnel diffraction. An example of this effect has been given in $[5,41]$.

As it has been discussed in Section 4.1 the interaction between excited quasi-molecular states not only produces spectral structures but also leads to nonadiabatic transitions, governed, in fact, by the same Massey parameter [3-6]. In the case under discussion, it leads to a connection between the spectral features and the cross sections of charge exchange in excited states. As an example inspired by the experiments [40], Figure 9 depicts the results of the closecoupled calculations of charge-exchange cross-sections in collisions $A l^{+12}(n=4)+C^{+6} \rightarrow A l^{+13}+C^{+5}(n=2)[42]$. As one can see for this reaction, the only $5 g \sigma-6 h \sigma$ channel accounts for charge exchange.

\section{Conclusion}

The goal of our consideration has been to clarify for ionic collisions the connection between nonelastic transitions and spectral profiles; this topic is well developed earlier in neutrals. The most distinctive collisions process involving ions is charge-exchange so that QM optical transitions have been analyzed in connection with charge-exchange process. Three typical cases have been considered, symmetric resonance charge-exchange, nonsymmetric charge-exchange involving accidental resonance, and charge-exchange in excited states with small energy defects. In all three cases, pronounced spectral structures have been exhibited. Therefore, the study of spectral profile features is intimately connected with the study of collision dynamics of ions in excited states. It is obvious that the existence of the connections between charge-exchange and spectral structures is a promising tool for plasma spectroscopy. Although the results obtained can be used directly for single collisions and low-density plasmas, they can be regarded as a reasonable starting approach and the zero-order approximation for laboratory and astrophysics hot dense plasma spectroscopy.

It has turned out that the study of the highly charged $Z_{1} e Z_{2}$ quasi-molecule forms a foolproof and fruitful approach for analyzing different features of spectral profiles and charge-exchange processes. The main result enhanced here is that the basic characteristics of QM, energy terms and dipole moments, can be obtained in the frame of the nonrelativistic Schrödinger equation, which is justified for low and moderate $Z$, with any desired accuracy. Following this approach we have carefully studied the general features of the dipole moments including the roots and the extrema, the reasons, and the features of the QM asymptotically forbidden transitions. The results obtained make clear that dipole transition moments are as equally important in calculations of spectral profiles as energy terms. It must be underlined that the fundamental $Z_{1} e Z_{2}$ problem, with its wide history [22], is of primary interest for atomic physics. So, the results discussed above can be regarded as a contribution to the study of optical characteristics of the $Z_{1} e Z_{2}$ quasi-molecule.

Finally, we summarize the highlights in the paper. It has been demonstrated that the existence of an extremum in the difference potential energy terms is not the necessary condition for a satellite in the spectral profiles. A root of the dipole moment has transformed a maximum in the profile into a dip. A global approximation has been proposed for the first time. The approximation is valid for the central part and the wings of the profiles as well as for the region of oscillations produced by extrema in the potential energy terms differences. As this study does not include the effect of the surrounding plasma on the quasi-molecule, it must be 
considered as a first step that needs to be improved, in order to be quantitatively used in plasma experiments.

It seems reasonable that the future developments of the physics of QM optical transitions will include specific calculations motivated by experiments in different kinds of plasmas as well as the study of the influence of external fields and environments. The investigations of dependencies of spectral features on external fields, temperature and density of charged particles are of the primary interest for diagnostics applications and for comparisons with experiments.

\section{Acknowledgment}

This research is supported by INTAS (Grant no. 06-10000179041) A. Derdariani would like also to gratefully acknowledge the hospitality of Professor E. Dalimier during his stay at Université Pierre et Marie Curie in 2009.

\section{References}

[1] E. E. Nikitin and S. Ya. Umanskii, Theory of Slow Atomic Collisions, vol. 30 of Series in Chemical Physics, Springer, Berlin, Germany, 1984.

[2] B. M. Smirnov, Plasma Processes and Plasma Kinetics: 586 Worked Out Problems for Science and Technology, chapter 3, Wiley-VCH, Weinheim, Germany, 2007.

[3] A. Devdariani, V. N. Ostrovskii, and Yu. N. Sebyakin, Soviet Technical Physics Letters, vol. 3, no. 9, p. 354, 1977.

[4] A. Devdariani, V. N. Ostrovskii, and Yu. N. Sebyakin, "Characteristics of electron and photon spectra associated with interaction between quasi-stationary states," Soviet Physics, vol. 49, no. 2, p. 266, 1979.

[5] A. Devdariani and Yu. N. Sebyakin, "Termperature dependence of the shape of the Landau-Zener satellite of a spectral line shape," Optics and Spectroscopy, vol. 48, no. 5, p. 557, 1980.

[6] A. Devdariani and Yu. N. Sebyakin, "Ionization with the participation of excited atoms," Soviet Physics, vol. 69, no. 7, p. 1127, 1989.

[7] M. J. O'Callaghen, A. Gallagher, and T. Holstein, "Absorption and emission of radiation in the region of an avoided level crossing," Physical Review A, vol. 32, no. 5, pp. 2754-2768, 1985.

[8] R. Beuc and V. Horvatic, "The investigation of the satellite rainbow in the spectra of diatomic molecules," Journal of Physics B, vol. 25, p. 1497, 1992.

[9] A. Gallagher, "Line shapes and radiation transfer," in Atomic, Molecular, \& Optical Physics, G. W. F Drake, Ed., pp. 220-232, Springer, New York, NY, USA, 2006.

[10] E. Dalimier, A. Poquérusse, and P. Angelo, "Space-resolved Xray emission from the densest part of laser plasmas: molecular satellite features and asymmetrical wings," Physical Review E, vol. 47, no. 3, pp. R1467-R1470, 1993.

[11] E. Oks and E. Dalimier, "Extrema in transition energies resulting not in satellites but in dips within spectral lines," Physical Review E, vol. 62, no. 3, pp. R3067-R3070, 2000.

[12] E. Oks and E. Dalimier, "Spectroscopic signatures of avoided crossings caused by charge exchange in plasmas," Journal of Physics B, vol. 33, no. 18, pp. 3795-3806, 2000.

[13] E. Dalimier, E. Oks, E. Dufour, et al., "Experimental discovery of charge-exchange-caused dips in spectral lines from laserproduced plasmas," Physical Review E, vol. 64, no. 6, Article ID 065401, 4 pages, 2001.
[14] E. Dalimier, E. Oks, E. Dufour, et al., "Advanced simulations of spectroscopic signatures of charge exchange in laser-produced plasmas," European Physical Journal D, vol. 20, no. 2, pp. 269$274,2002$.

[15] J. B. Greenwood, I. D. Williams, S. J. Smith, and A. Chutjian, "Experimental investigation of the processes determining Xray emission intensities from charge-exchange collisions," Physical Review A, vol. 63, no. 6, Article ID 062707, 9 pages, 2001.

[16] F. B. Rosmej, D. Reiter, V. S. Lisitsa, et al., "Influence of chargeexchange processes on X-ray spectra in TEXTOR tokamak plasmas: experimental and theoretical investigation," Plasma Physics and Controlled Fusion, vol. 41, no. 2, pp. 191-214, 1999.

[17] R. Anholt, "X rays from quasimolecules," Reviews of Modern Physics, vol. 57, no. 4, pp. 995-1053, 1985.

[18] K. Schulze, J. Anton, T. Jacob, W.-D. Sepp, and B. Fricke, "Quasimolecular X rays in the $\mathrm{Cl}^{16+}-\mathrm{Ar}$ collision system," Physical Review A, vol. 63, no. 2, Article ID 022503, 8 pages, 2001.

[19] P. Gauthier, S. J. Rose, P. Sauvan, et al., "Modeling the radiative properties of dense plasmas," Physical Review E, vol. 58, no. 1, pp. 942-950, 1998.

[20] P. Sauvan, P. Angelo, A. Calisti, E. Dalimier, and E. Minguez, "Improving the dicenter model for hot dense plasmas: molecular stark effect," Journal of Quantitative Spectroscopy and Radiative Transfer, vol. 71, no. 2-6, pp. 675-687, 2001.

[21] A. Devdariani, T. M. Kereselidze, I. L. Noselidze, et al., "Dipole transition-matrix elements of the one-electron heterodiatomic quasimolecules," Physical Review A, vol. 71, no. 2, Article ID 022512, 2005.

[22] I. V. Komarov, L. I. Ponomarev, and S. Yu. Slavyanov, Spheroidal and Coulomb Spheroidal Functions, Nauka, Moscow, Russia, 1976.

[23] I. I. Sobel'man, Introduction to the Theory of Atomic Spectra, Pergamon Press, Oxford, UK, 1972.

[24] K. Taulbjerg, J. Vaaben, and B. Fastrup, "Molecular-orbital theory of K-vacancy sharing in atomic collisions," Physical Review A, vol. 12, p. 2325, 1975.

[25] E. E. Nikitin and S. Ya. Umanskii, Theory of Slow Atomic Collisions, Springer, New York, NY, USA, 1984.

[26] A. Devdariani, in Spectral Line Shapes, R. Stamm and B. Talin, Eds., vol. 7, p. 235, Nova, Hauppauge, NY, USA, 1993.

[27] A. Devdariani, "Emission of quasi-molecules," Optics and Spectroscopy, vol. 86, no. 6, pp. 853-858, 1999.

[28] E. Bichoutskaia, A. Devdariani, K. Ohmori, O. Misaki, K. Ueda, and Y. Sato, "Spectroscopy of quasimolecular optical transitions: $\mathrm{Ca}\left(4 \mathrm{~s}^{21} \mathrm{~S}_{0} \leftrightarrow 4 \mathrm{~s} 4 \mathrm{p}^{1} \mathrm{P}, 4 \mathrm{~s} 3 \mathrm{~d}^{1} \mathrm{D}_{2}\right)-\mathrm{He}$. The influence of radiation width," Journal of Physics B, vol. 34, no. 12, pp. 2301-2312, 2001.

[29] A. Devdariani, E. Dalimier, P. Sauvan, and P. Angelo, "Influence of the exchange interaction on Ly- $\alpha$ radiation of multicharged ions," Physical Review A, vol. 66, no. 5, Article ID 052703, 4 pages, 2002.

[30] A. Devdariani, T. Kereselidze, I. Noselidze, et al., "Optical transitions in one-electron quasi-molecules. The influence of the R-dependent dipole transition moments and the trajectories," in Proceedings of the 25th International Conference on Photonic, Electronic and Atomic Collisions (ICPEAC '07), Freiburg, Germany, July 2007, Mo085.

[31] D. E. Remaker and J. M. Peek, " ${ }^{2} \mathrm{H}_{2}^{+}$dipole strengths by asymptotic techniques," Journal of Physics B: Atomic, Molucular, and Optical Physics, vol. 5, p. 2175, 1972.

[32] D. E. Ramaker and J. M. Peek, "Dipole strengths involving the lowest twenty electronic states of $\mathrm{H}_{2}^{+}$," Atomic Data and Nuclear Data Tables, vol. 5, no. 2, pp. 167-184, 1973. 
[33] N. F. Allard, J. F. Kielkopf, G. Hébrard, and J. M. Peek, "Theoretical study of the Lyman $\gamma$ line profile of atomic hydrogen perturbed by collisions with protons. Lyman $\gamma$ line profile," European Physical Journal D, vol. 29, no. 1, pp. 7-16, 2004.

[34] A. Devdariani and E. Dalimier, "Radiative characteristics of quasimolecular state interactions," Physical Review A, vol. 78, Article ID 022512, 2008.

[35] A. Devdariani, Rapport Scientifique, Laboratoire pour l'Utilisation des Lasers Intenses, CNRS-Ecole PolytechniqueUniversite Paris VI, Paris, France, 1995.

[36] N. Allard and J. Kielkopf, "The effect of neutral nonresonant collisions on atomic spectral lines," Reviews of Modern Physics, vol. 54, no. 4, pp. 1103-1182, 1982.

[37] B. M. Smirnov, Asymptotic Methods in Atomic Collision Theory, Atomizdat, Moscow, Russia, 1973.

[38] V. A. Astapenko and V. S. Lisitsa, "Effect of charge exchange on atomic spectral line shapes in plasmas: asymptotic theory," in Spectral Line Shapes, J. Seidel, Ed., pp. 147-149, American Institute of Physics, 2001.

[39] A. Devdariani, E. Bichoutskaia, E. Tchesnokov, et al., "Semiclassical analytical approach to the description of quasimolecular optical transitions," Journal of Physics B, vol. 35, no. 11, pp. 2469-2475, 2002.

[40] E. Dalimier, E. Oks, O. Renner, and R. Schott, "Experimental determination of rate coefficients of charge exchange from $\mathrm{x}$ dips in laser-produced plasmas," Journal of Physics B, vol. 40, no. 5, pp. 909-919, 2007.

[41] A. Devdariani, V. N. Ostrovskii, I. K. Ryzhikova, and Yu. N. Sebyakin, Vestnik LGU, no. 22, p. 36, 1978.

[42] A. Devdariani, E. Dalimier, T. Kereselidze, I. Noselidze, F. Rebentrost, and P. Sauvan, "Characteristics of quasi-molecular state interactions," in Proceedings of the Spectral Line Shapes: Volume 15-19th International Conference on Spectral Line Shapes, M. A. Gigosos and M. Á. González, Eds., vol. 1058 of AIP Conference Proceedings, pp. 102-115, 2008. 


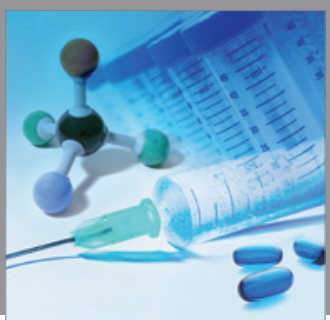

International Journal of

Medicinal Chemistry

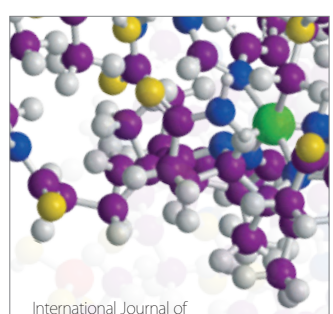

Carbohydrate Chemistry

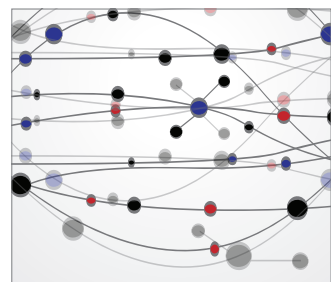

The Scientific World Journal
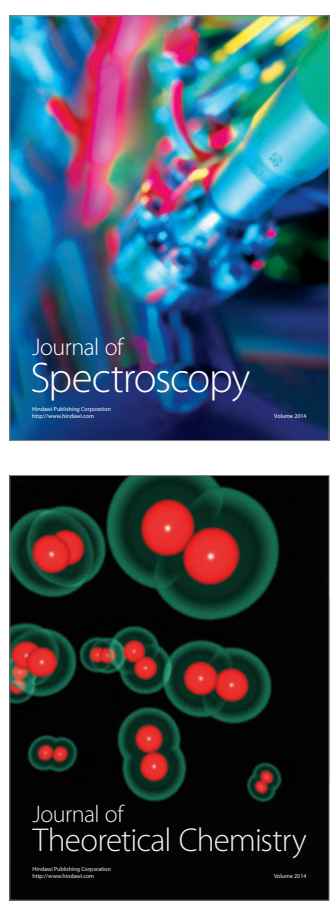
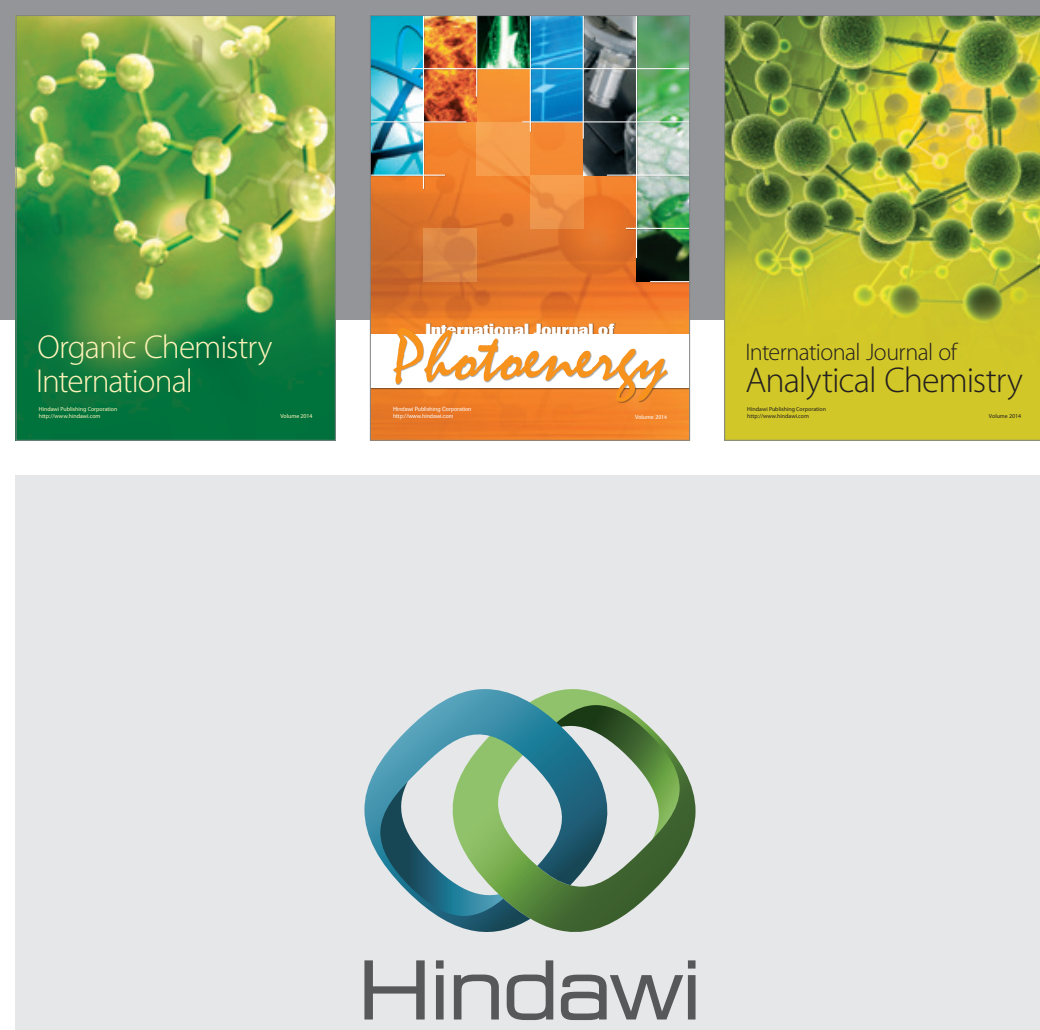

Submit your manuscripts at

http://www.hindawi.com
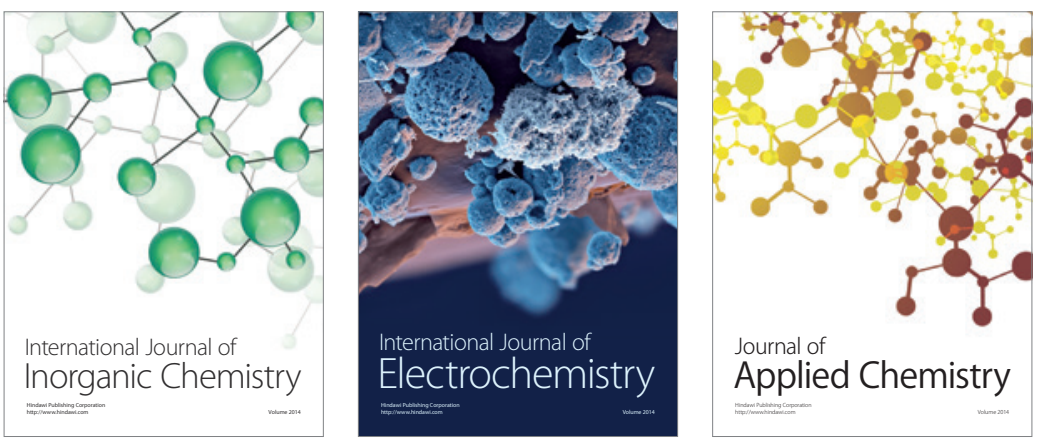

Journal of

Applied Chemistry
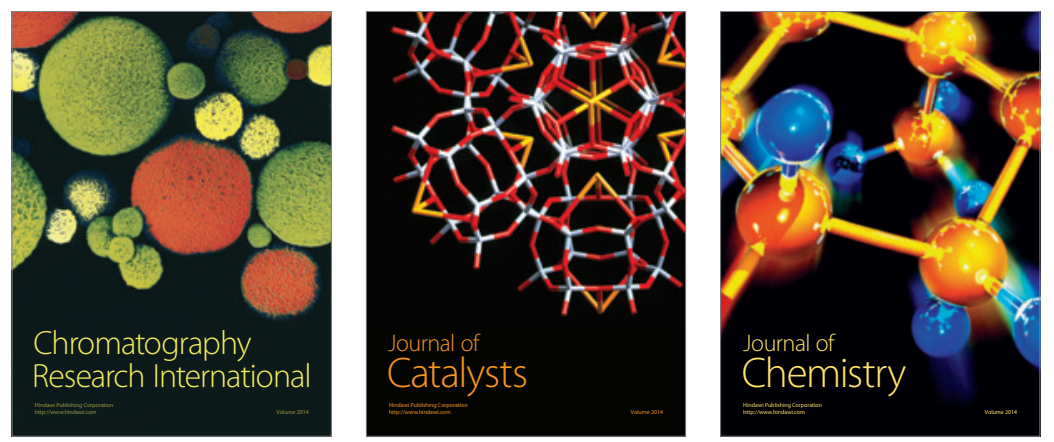
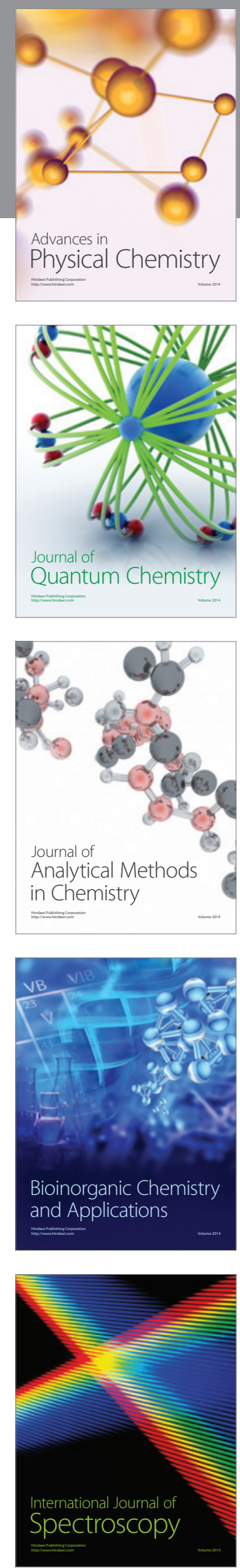